\title{
Point defect approach to enhance the thermoelectric performance of Zintl-phase BaAgSb
}

\author{
Yifang Huang ${ }^{1 \dagger}$, Chen Chen ${ }^{1,2 \dagger}$, Weiming Zhang ${ }^{1}$, Xiaofang $\mathrm{Li}^{1}$, Wenhua Xue ${ }^{1,3}$, Xinyu Wang ${ }^{1}$, \\ Yijie Liu ${ }^{4}$, Honghao Yao ${ }^{1}$, Zongwei Zhang ${ }^{1}$, Yue Chen ${ }^{2 *}$, Feng Cao ${ }^{4 *}$, Xingjun Liu ${ }^{1,5}$, \\ Yumei Wang ${ }^{3}$ and Qian Zhang ${ }^{1,5^{*}}$
}

\begin{abstract}
Zintl-phase compounds have great potential in thermoelectric applications owing to their "phonon glasselectron crystal" (PGEC) structures. In this paper, a new Zintlphase thermoelectric material $\mathrm{BaAgSb}$ is reported. Ba deficiency increased the carrier concentration, and then suppressed the intrinsic excitation. The peak $Z T$ value of $\mathrm{Ba}_{0.98} \mathrm{AgSb}$ reached $\sim 0.56$ at $773 \mathrm{~K}$. Moreover, Eu alloying at Ba site not only lowered the lattice thermal conductivity by inducing point-defect scattering, but also improved the electrical properties by increasing the carrier mobility. Finally, a peak $Z T$ of $\sim 0.73$ was achieved in $\mathrm{Ba}_{0.78} \mathrm{Eu}_{0.2} \mathrm{AgSb}$.
\end{abstract}

Keywords: thermoelectric, Zintl phase, point defect, isoelectronic alloying

\section{INTRODUCTION}

Various energy conversion technologies have been suggested to reduce the consumption of non-renewable resources, and promote the sustainable development of global community. Thermoelectric materials, which can realize the mutual conversion between electricity and thermal energy, have a great prospect in waste heat recovery and solid-state refrigeration $[1,2]$. However, the development of devices made of thermoelectric materials is still limited by the energy conversion efficiency that requires a high figure-of-merit $Z T=S^{2} \sigma T / \kappa$, where $S, \sigma, \kappa$ and $T$ are Seebeck coefficient, electrical conductivity, thermal conductivity, and absolute temperature, respectively [3]. To achieve high thermoelectric performance, excellent electrical properties and low thermal conductivity are necessary. Up to now, band engineering $[4,5]$, point defects engineering [6,7], nanoscale engineering $[8,9]$, entropy engineering $[10,11]$, phase diagram engineering $[12,13]$, etc. have proved effective in optimizing thermoelectric properties.

Zintl-phase compounds are promising thermoelectric materials with typical "phonon glass-electron crystal" (PGEC) structure [14,15]. Point defects mainly caused by dopants, vacancies, alloying, and interstitials are easily introduced in this kind of compounds, because of the complex structure and rich component elements $[14,16]$. The dopant or interstitial atoms can regulate the Fermi level by tuning the carrier concentration, and the alloying atoms can optimize the band structure by orbital alignment for the improvement of the electrical transport properties [17-21]. In addition, the guest atoms or vacancies can suppress the lattice thermal conductivity by strengthening phonon scattering [22-24]. Indeed, high thermoelectric efficiency has been realized in some Zintl phases, such as $\mathrm{YbZn}_{2} \mathrm{Sb}_{2}[25,26], \mathrm{Ca}_{9} \mathrm{Zn}_{4.5} \mathrm{Sb}_{9}[27,28]$, $\mathrm{Ca}_{5} \mathrm{Al}_{2} \mathrm{Sb}_{6}$ [29], and $\mathrm{Yb}_{14} \mathrm{MnSb}_{11}$ [30]. Our previous studies reported the thermoelectric performance of the ZrBeSi-type Zintl-phase $\mathrm{Eu}_{2} \mathrm{ZnSb}_{2}$, and a $Z T$ value of 1.0 was achieved at $823 \mathrm{~K}$ [31]. Different from $\mathrm{Eu}_{2} \mathrm{ZnSb}_{2}$, $\mathrm{SrAgSb}$ and EuAgSb possess more attractive electrical transport properties due to the fully occupied framework [32-34]. As a typical ZrBeSi-type Zintl compound, $\mathrm{BaAgSb}$ may also have promising thermoelectric perfor-

\footnotetext{
${ }^{1}$ School of Materials Science and Engineering, and Institute of Materials Genome \& Big Data, Harbin Institute of Technology, Shenzhen 518055, China

${ }^{2}$ Department of Mechanical Engineering, The University of Hong Kong, Pokfulam Road, Hong Kong SAR, China

${ }^{3}$ Beijing National Laboratory for Condensed Matter Physics, Institute of Physics, Chinese Academy of Sciences, Beijing 100190, China

${ }^{4}$ School of Science, Harbin Institute of Technology, Shenzhen 518055, China

${ }^{5}$ State Key Laboratory of Advanced Welding and Joining, Harbin Institute of Technology, Harbin 150001, China

${ }^{\dagger}$ These authors contributed equally to this work.

* Corresponding authors (emails: yuechen@hku.hk (Chen Y); caofeng@hit.edu.cn (Cao F); zhangqf@hit.edu.cn (Zhang Q))
} 
mance after coordinate regulation of electrical and phononic properties.

In this work, Zintl-phase BaAgSb was prepared by high-energy ball milling followed by spark plasma sintering (SPS). The intrinsic excitation inhibited the thermoelectric performance of $\mathrm{BaAgSb}$ at high temperatures. In order to optimize the thermoelectric properties of $\mathrm{BaAgSb}$, two types of point defects were introduced. Firstly, Ba deficiency introduced more hole carriers, and suppressed the intrinsic excitation. Benefiting from the improved electrical properties, a $Z T$ value of $\sim 0.56$ was achieved in $\mathrm{Ba}_{0.98} \mathrm{AgSb}$ at $773 \mathrm{~K}$. Furthermore, $\mathrm{Ba}_{0.98-y} \mathrm{Eu}_{y} \mathrm{AgSb}(y=0.1,0.2$, and 0.3$)$ were designed to regulate the thermoelectric properties. The reduction in the lattice thermal conductivity was achieved through alloying $\mathrm{Eu}$ at the $\mathrm{Ba}$ site. In addition, Eu alloying brought about both the increased carrier concentration and mobility. As a result, maximum power factor and $Z T$ reached respectively $\sim 1.2 \times 10^{-3} \mathrm{~W} \mathrm{~m}^{-1} \mathrm{~K}^{-2}$ and $\sim 0.73$ at $773 \mathrm{~K}$ for $\mathrm{Ba}_{0.78} \mathrm{Eu}_{0.2} \mathrm{AgSb}$.

\section{EXPERIMENTAL SECTION}

\section{Synthesis}

Barium (Ba, 99.9\%, pieces), silver (Ag, 99.9\%, powder), antimony ( $\mathrm{Sb}, 99.999 \%$, shots), and europium (Eu, $99.9 \%$, chunk) were weighed according to the nominal compositions of $\mathrm{Ba}_{1-x} \mathrm{AgSb}(x=0,0.01,0.02$, and 0.03$)$ and $\mathrm{Ba}_{0.98-y} \mathrm{Eu}_{y} \mathrm{AgSb}(y=0.1,0.2$, and 0.3$)$, and loaded into a stainless-steel jar with stainless-steel balls in a glove box. The mixtures were ball-milled continuously for $10 \mathrm{~h}$ using a high energy ball mill (SPEX $8000 \mathrm{M})$. The obtained powder was sintered into a dense disk at $923 \mathrm{~K}$ for 2 min by SPS under a pressure of $60 \mathrm{MPa}$.

\section{Characterization}

The crystal structures were studied by X-ray diffraction (XRD, Rigaku D/max 2500 PC). The microstructures were investigated by a spherical aberration-corrected (Cscorrected) electron microscope (JEM-ARM200F). The electrical conductivity and Seebeck coefficient were measured on a commercial apparatus (CTA-3, Cryoall). The uncertainty for the electrical conductivity and the Seebeck coefficient are 3\% and 5\%, respectively. The thermal conductivity $(\kappa)$ was calculated using $\kappa=D C_{\mathrm{p}} \alpha$, where $D$ is the volume density determined by the Archimedes method (shown in Table S1), $C_{\mathrm{p}}$ is the specific heat estimated by a differential scanning calorimetry thermal analyzer (Netzsch DSC 404F3), and $\alpha$ is the thermal diffusivity measured on a laser flash apparatus
(Netzsch LFA 457). The uncertainty for the thermal conductivity is $6 \%$ (comprising uncertainties of $4 \%$ for the thermal diffusivity, $3.5 \%$ for the specific heat, and $3 \%$ for the density). The temperature-dependent Hall coefficient $\left(R_{\mathrm{H}}\right)$ was measured based on the van-der-Pauw technique under a reversible magnetic field of $1.5 \mathrm{~T}$. The Hall carrier concentration $\left(n_{\mathrm{H}}\right)$ and Hall mobility $\left(\mu_{\mathrm{H}}\right)$ were calculated using $n_{\mathrm{H}}=1 /\left(e R_{\mathrm{H}}\right)$ and $\mu_{\mathrm{H}}=\sigma R_{\mathrm{H}}$, respectively. The relation of Kubelka-Munk function $(F(R))$ versus wavelength $(\lambda)$ was obtained by a Nicolet IS50 FTIR spectrophotometer. For the indirect transition, the optical band gap $\left(E_{\mathrm{g}}\right)$ was determined based on the relational expression $\left(F(R)^{*} h v\right)^{0.5}=B\left(h v-E_{\mathrm{g}}\right)$, where $h$ is the Planck constant, and $v$ is the frequency of light.

\section{Calculation}

The density functional theory (DFT) calculations were performed using the Vienna $A b$-initio Simulation Package (VASP). The generalized gradient approximation (GGA) of the Perdew-Burke-Ernzerhof (PBE) functional with the projector-augmented wave (PAW) method was used during the calculations of the total energies, band structure, and the geometry relaxations $[35,36]$. The total energy and Hellmann-Feynman force convergence criteria were $0.1 \mathrm{meV}$ atom $^{-1}$ and $0.01 \mathrm{eV} \AA^{-1}$, respectively. A plane wave cutoff energy of $400 \mathrm{eV}$ and a Gamma centered $k$-mesh $6 \times 6 \times 4$ were chosen for the crystal structure relaxation.

\section{RESULTS AND DISCUSSION}

ZrBeSi-type Zintl compounds with hexagonal structure (space group: $P 6_{3} / \mathrm{mmc}$ ) have been considered as promising mid-temperature thermoelectric materials $[16,31,33]$. Different from $\mathrm{ZrBeSi}$-type $\mathrm{Eu}_{2} \mathrm{ZnSb}_{2}$ [31] and $\mathrm{Eu}_{2} \mathrm{ZnBi}_{2}$ [37], which have $50 \%$ vacancies at the $\mathrm{Zn}$ sites, the $\mathrm{Ag}$ sites are $100 \%$ occupied in $\mathrm{BaAgSb}$, as shown in Fig. 1a, b. This fully occupied anionic framework benefits the carrier transport. Fig. 1c shows the electron localization function (ELF) of BaAgSb with the slice parallel to the (110) plane. ELF provides a good description of electron delocalization, which can be used to well understand the Zintl structure. The values of 1 and 0 correspond to fully localized and very low charge density, respectively [38]. The overlap of ELF between Ag and Sb suggests that they are connected by strong chemical bonds. The low charge density between $\mathrm{Ba}^{2+}$ and $[\mathrm{AgSb}]^{2-}$ framework indicates that ionic bond exists in the $\mathrm{BaAgSb}$ Zintl phase. We also calculated the band structure of $\mathrm{BaAgSb}$ using density functional method and presented the results in Fig. 1d. The calculated band gap of BaAgSb 


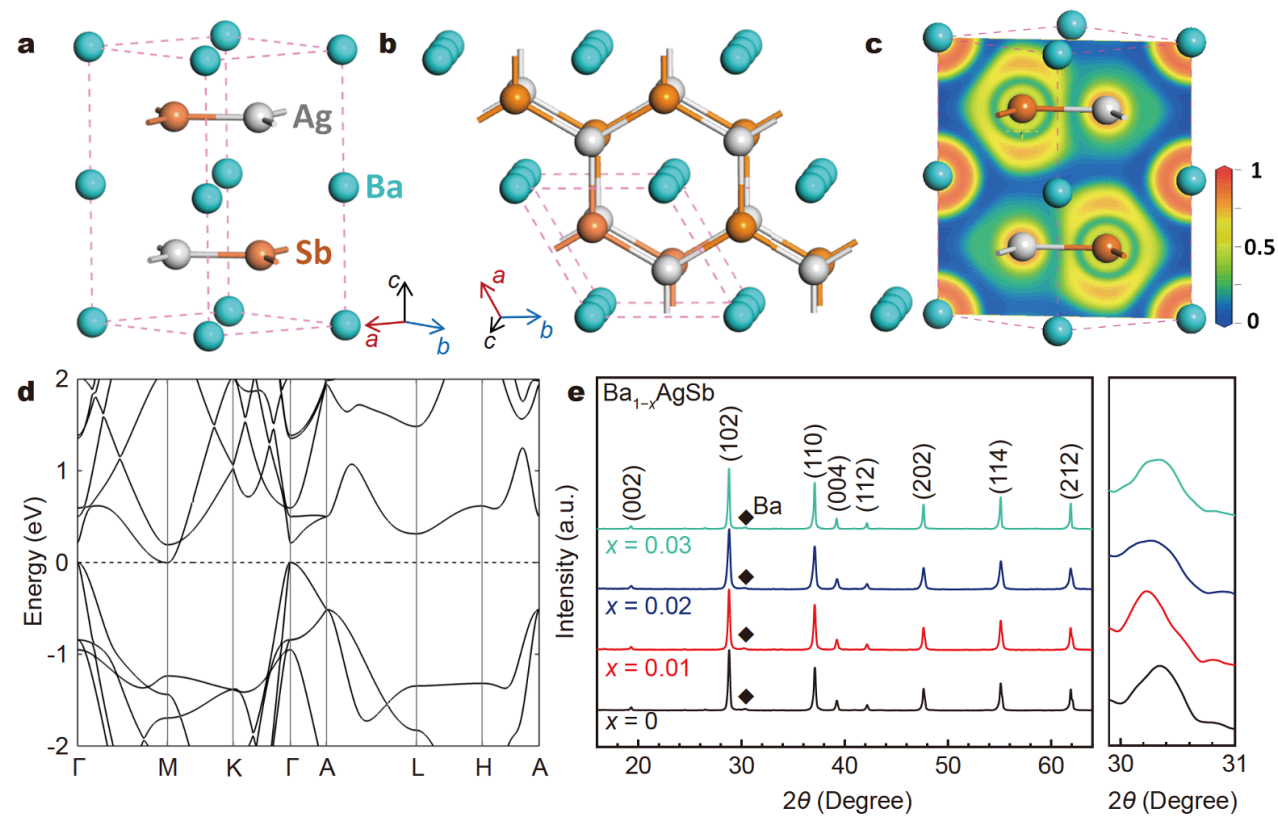

Figure 1 Crystal structure of Zintl-phase BaAgSb viewed from (a) [110] and (b) [001] direction. The cyan, gray, and golden spheres represent the barium, silver, and antimony, respectively. (c) Electron localization function (ELF) of BaAgSb. (d) Calculated band structure of BaAgSb. (e) XRD patterns of $\mathrm{Ba}_{1-x} \mathrm{AgSb}(x=0,0.01,0.02$, and 0.03$)$. The patterns from $30^{\circ}$ to $31^{\circ}$ are enlarged.

is near $0 \mathrm{eV}$. Considering that PBE usually underestimates experimental band gap, the BaAgSb might be a semiconductor with a narrow band gap, which is similar with SrAgSb and EuAgSb [33]. The conduction band minimum (CBM) locates around the $M$ point, while the valence band maximum (VBM) locates around the $\Gamma$ point. Two hybrid bands are observed near the band edge of the valence band without obvious energy difference, which is good for the electrical properties [39]. By directly ball milling and hot pressing, a series of samples with different contents of $\mathrm{Ba}$ in $\mathrm{Ba}_{1-x} \mathrm{AgSb}(x=0,0.01,0.02$, and 0.03) were prepared. As shown in Fig. 1e, the main peaks of BaAgSb match well with the standard diffraction pattern; however, a little amount of impurity $\mathrm{Ba}$ is also observed. The peak of this secondary phase is weakened with the decrease of the Ba content.

Temperature-dependent electrical conductivity and Seebeck coefficient of $\mathrm{Ba}_{1-x} \mathrm{AgSb}(x=0,0.01,0.02$, and 0.03 ) are shown in Fig. 2a, b, respectively. The electrical conductivity increases and the Seebeck coefficient decreases with the reduced $\mathrm{Ba}$ content because of the increased carrier concentration caused by Ba deficiency at temperatures below $550 \mathrm{~K}$. Due to the onset of the intrinsic excitation at temperatures higher than $550 \mathrm{~K}$, the Seebeck coefficient decreases significantly, causing a reduced power factor of BaAgSb, as shown in Fig. 2c. With decreasing content of $\mathrm{Ba}$ and then increasing carrier concentration, the intrinsic excitation is suppressed, contributing to an enhanced power factor at high temperatures. The maximum power factor of $\mathrm{Ba}_{0.98} \mathrm{AgSb}$ reaches $\sim 1.0 \times 10^{-3} \mathrm{~W} \mathrm{~m}^{-1} \mathrm{~K}^{-2}$ at $773 \mathrm{~K}$, which is higher than those of $\mathrm{Eu}_{2} \mathrm{ZnSb}_{2}$-based Zintl compounds [34,37]. The temperature-dependent carrier concentration and mobility are plotted in Fig. 2d. With the introduction of Ba deficiency, the intrinsic excitation moves to higher temperatures. Meanwhile, the increased carrier concentration strengthens the carrier scattering and leads to decreased carrier mobility.

The thermal conductivity and $Z T$ values of $\mathrm{Ba}_{1-x} \mathrm{AgSb}$ $(x=0,0.01,0.02$, and 0.03$)$ are presented in Fig. 3. The thermal conductivity of $\mathrm{Ba}_{1-x} \mathrm{AgSb}$ displays a decreasing trend with increasing temperature. Compared with the electrical properties, Ba deficiency brings about a small impact on the thermal conductivity. The slightly increased thermal conductivities of $\mathrm{Ba}_{0.98} \mathrm{AgSb}$ and $\mathrm{Ba}_{0.97} \mathrm{AgSb}$ are mainly related to the increased carrier contribution. The lattice thermal conductivity $\kappa_{\mathrm{L}}$ was obtained by subtracting the carrier contribution $\kappa_{\mathrm{c}}$ from the total thermal conductivity $\kappa$. The $\kappa_{\mathrm{c}}$ was estimated using the Wiedemann-Franz relationship $\left(\kappa_{\mathrm{c}}=L \sigma T\right)$, where $L$ is the Lorenz number and calculated using a single parabolic band (SPB) model $[19,22,33]$. To be 

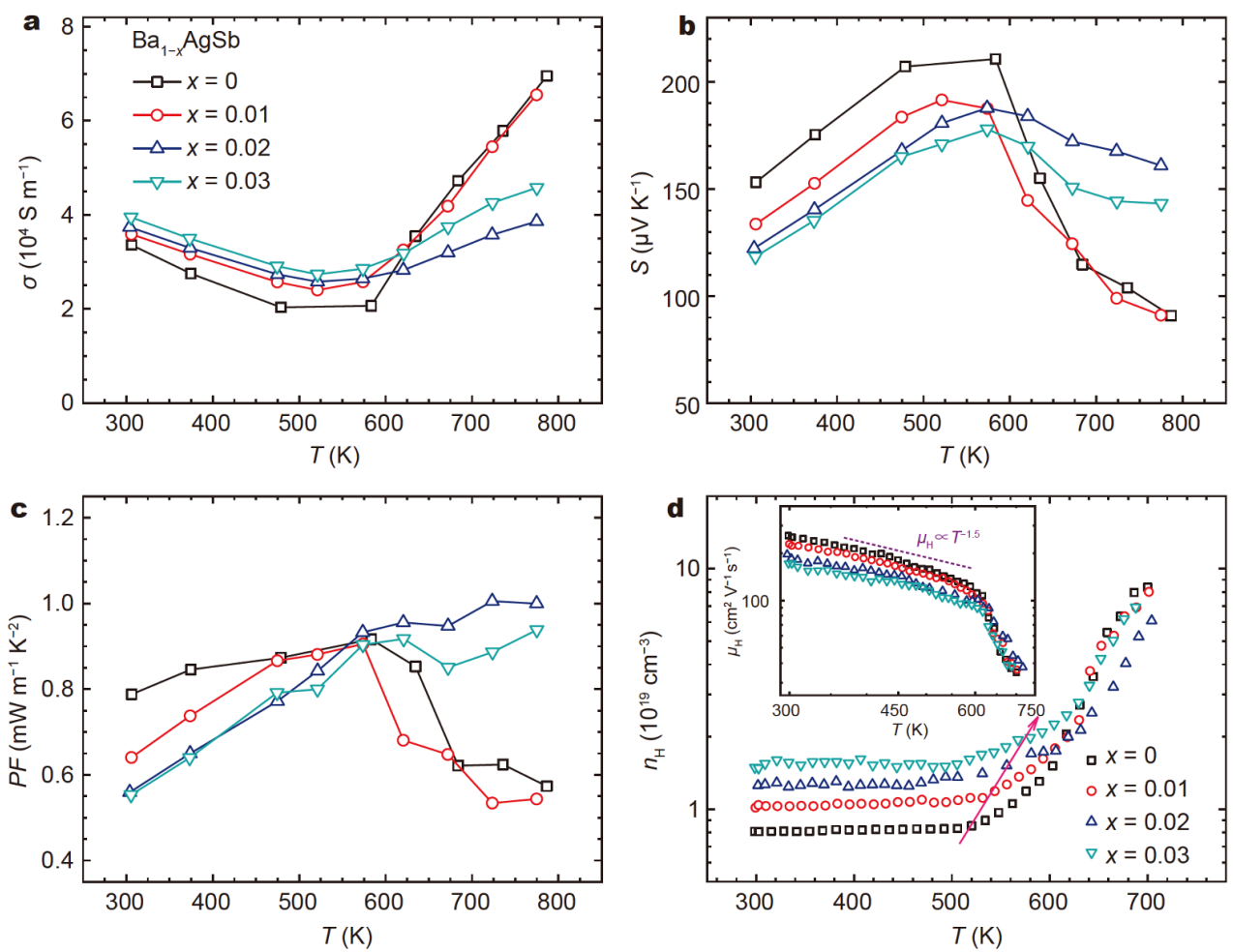

Figure 2 Temperature-dependent (a) electrical conductivity, (b) Seebeck coefficient, and (c) power factor of $\mathrm{Ba}_{1-x} \mathrm{AgSb}(x=0,0.01,0.02$, and 0.03$)$. (d) $n_{\mathrm{H}}$ and mobility of $\mathrm{Ba}_{1-x} \mathrm{AgSb}(x=0,0.01,0.02$, and 0.03$)$ as a function of temperature.
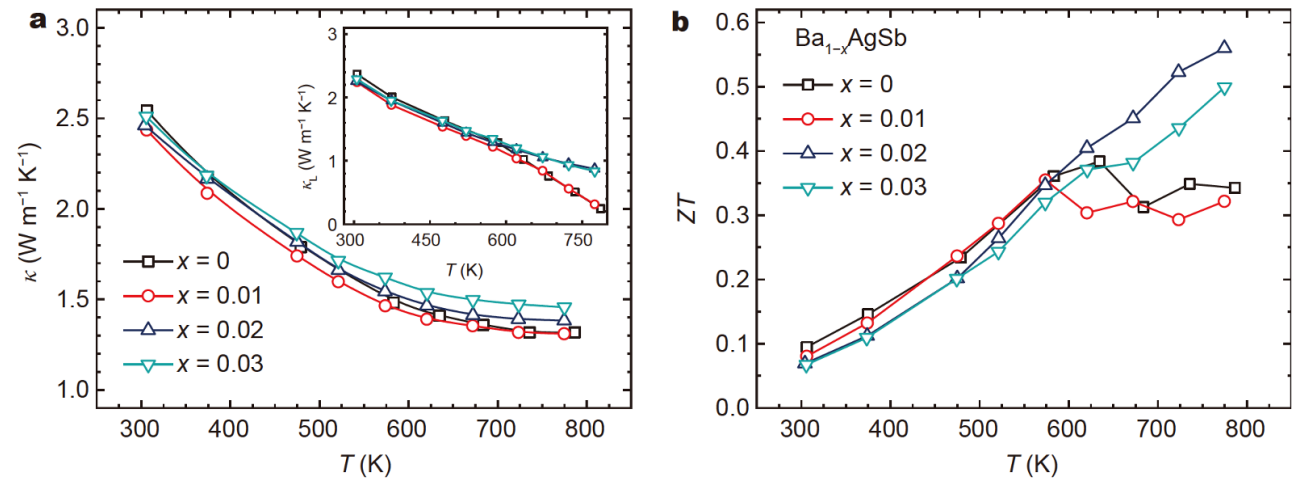

Figure 3 (a) Temperature-dependent thermal conductivity of $\mathrm{Ba}_{1-x} \operatorname{AgSb}(x=0,0.01,0.02$, and 0.03$)$. The inset shows the lattice thermal conductivity. (b) Temperature-dependent $Z T$ of $\mathrm{Ba}_{1-x} \mathrm{AgSb}(x=0,0.01,0.02$, and 0.03$)$.

noted, the difference of lattice thermal conductivity at high temperatures is probably due to the intrinsic excitation, which makes the SPB model not suitable [40]. Benefiting from the suppressed intrinsic excitation, the maximum $Z T$ value of $\mathrm{Ba}_{0.98} \mathrm{AgSb}$ reaches $\sim 0.56$ at $773 \mathrm{~K}$, which is higher than that of $\mathrm{BaAgSb}$.

Compared with $\mathrm{Eu}_{2} \mathrm{ZnSb}_{2}, \mathrm{BaAgSb}$ has better electrical properties and relatively higher thermal conductivity. To further enhance the thermoelectric properties, a series of samples $\mathrm{Ba}_{0.98-y} \mathrm{Eu}_{y} \mathrm{AgSb}(y=0.1,0.2$, and 0.3 ) were prepared by isoelectronic alloying $\mathrm{Eu}$ at the Ba site. As shown in Fig. $4 \mathrm{a}$, all the Eu-alloyed samples are single phase with $\mathrm{ZrBeSi}$ structure. The secondary phase Ba disappears after $\mathrm{Eu}$ alloying. The XRD peak positions move slightly to a larger angle with increasing Eu content because of the smaller atomic radius of Eu. The whole-pattern refined lattice parameters (displayed in Fig. 4b) decrease from $a=$ $b=4.847 \AA$ and $c=9.186 \AA$ for $\mathrm{Ba}_{0.98} \mathrm{AgSb}$ to $a=b=$ 

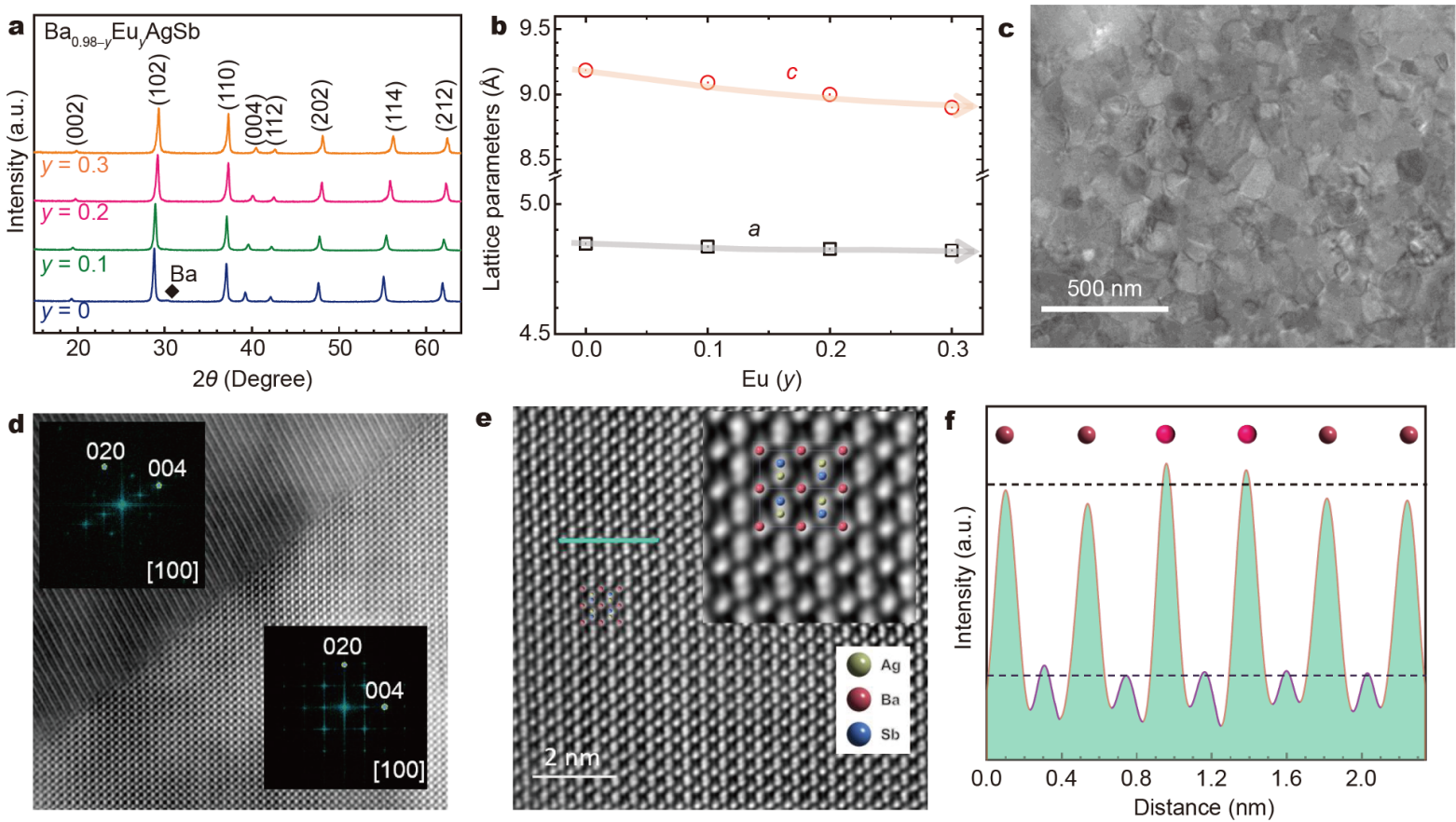

Figure 4 (a) XRD patterns and (b) refined lattice parameters of $\mathrm{Ba}_{0.98-y} \mathrm{Eu}_{y} \mathrm{AgSb}(y=0,0.1,0.2$, and 0.3). (c) Low-magnification TEM image of $\mathrm{Ba}_{0.78} \mathrm{Eu}_{0.2} \mathrm{AgSb}$. (d) STEM image of two contiguous grains. The insets in (d) are the SAED patterns taken along the [001] direction. (e) HAADF-STEM image along the [100] direction of $\mathrm{Ba}_{0.78} \mathrm{Eu}_{0.2} \mathrm{AgSb}$. (f) The intensity profile of different atoms along the aquamarine line in (e).

$4.821 \AA$ and $c=8.899 \AA$ for $\mathrm{Ba}_{0.68} \mathrm{Eu}_{0.3}$ AgSb. Fig. $4 \mathrm{c}$ shows the low-magnification transmission electron microscopy (TEM) image of the $\mathrm{Ba}_{0.78} \mathrm{Eu}_{0.2} \mathrm{AgSb}$ sample, which displays that the grain size is $\sim 200 \mathrm{~nm}$. Small grain size usually indicates that a lot of grain boundaries exist in the sample. The scanning TEM (STEM) image of two contiguous grains with clear grain boundary is presented in Fig. 4d. The corresponding selected area electron diffraction (SAED) pattern (insets of Fig. 4d) can be indexed along the [001] direction. We also estimated the angle between the two grains, which is about $28.3^{\circ}$. The Cscorrected high-angle annular dark-field STEM (HAADFSTEM) image of BaAgSb is shown in Fig. 4e. As is well known, the HAADF-STEM offers a unique technique to characterize the crystal structure. The atomic sites match well with the projection structure of $\mathrm{BaAgSb}$ along the [100] direction. Ba vacancies are difficult to be observed in the HAADF-STEM image, because the content of Ba deficiency is too small. The intensity of the atoms along the aquamarine line in Fig. 4e is plotted in Fig. 4f. Different from the conventional TEM images, the contrast of HAADF-STEM images is roughly proportional to $Z^{1.7}$ ( $Z$ is the atomic number). Six atoms at the Ba sites stand in the aquamarine line. Parts of atomic intensities at the Ba sites are higher than others. We believe that the Eu atoms partially occupy the Ba sites due to the larger atomic number of $\mathrm{Eu}(63)$ than that of $\mathrm{Ba}(56)$. The signals of $\mathrm{Ag}$ and $\mathrm{Sb}$ (purple peaks) are weak, because the aquamarine line does not cross directly above $\mathrm{Ag}$ and $\mathrm{Sb}$ atoms.

The temperature-dependent electrical conductivity and Seebeck coefficient of $\mathrm{Ba}_{0.98-y} \mathrm{Eu}_{y} \mathrm{AgSb}(y=0,0.1,0.2$, and 0.3) are shown in Fig. 5a, b, respectively. With increasing content of Eu, the electrical conductivity improves over the whole temperature range, while the Seebeck coefficient decreases after Eu alloying. The obviously improved electrical conductivity contributes to the enhanced power factor, presented in Fig. 5c. The maximum power factor reaches $\sim 1.2 \times 10^{-3} \mathrm{~W} \mathrm{~m}^{-1} \mathrm{~K}^{-2}$ at $\sim 773 \mathrm{~K}$ for $\mathrm{Ba}_{0.78} \mathrm{Eu}_{0.2^{-}}$ $\mathrm{AgSb}$ and $\mathrm{Ba}_{0.68} \mathrm{Eu}_{0.3} \mathrm{AgSb}$. Fig. $5 \mathrm{~d}$ shows the room-temperature Hall carrier concentration and mobility of $\mathrm{Ba}_{0.98-y} \mathrm{Eu}_{y} \mathrm{AgSb}(y=0.1,0.2$, and 0.3$)$. The increased electrical conductivity of Eu-alloyed samples is not only due to the increased carrier concentration but also the increased mobility.

Normally, the increased carrier concentration will aggravate the carrier scattering, and bring about the decreased mobility. To further clarify the electrical transport mechanism, high-temperature Hall measurement was conducted, and the results are presented in Fig. 6a, b. With the increase of Eu substitution, the carrier con- 

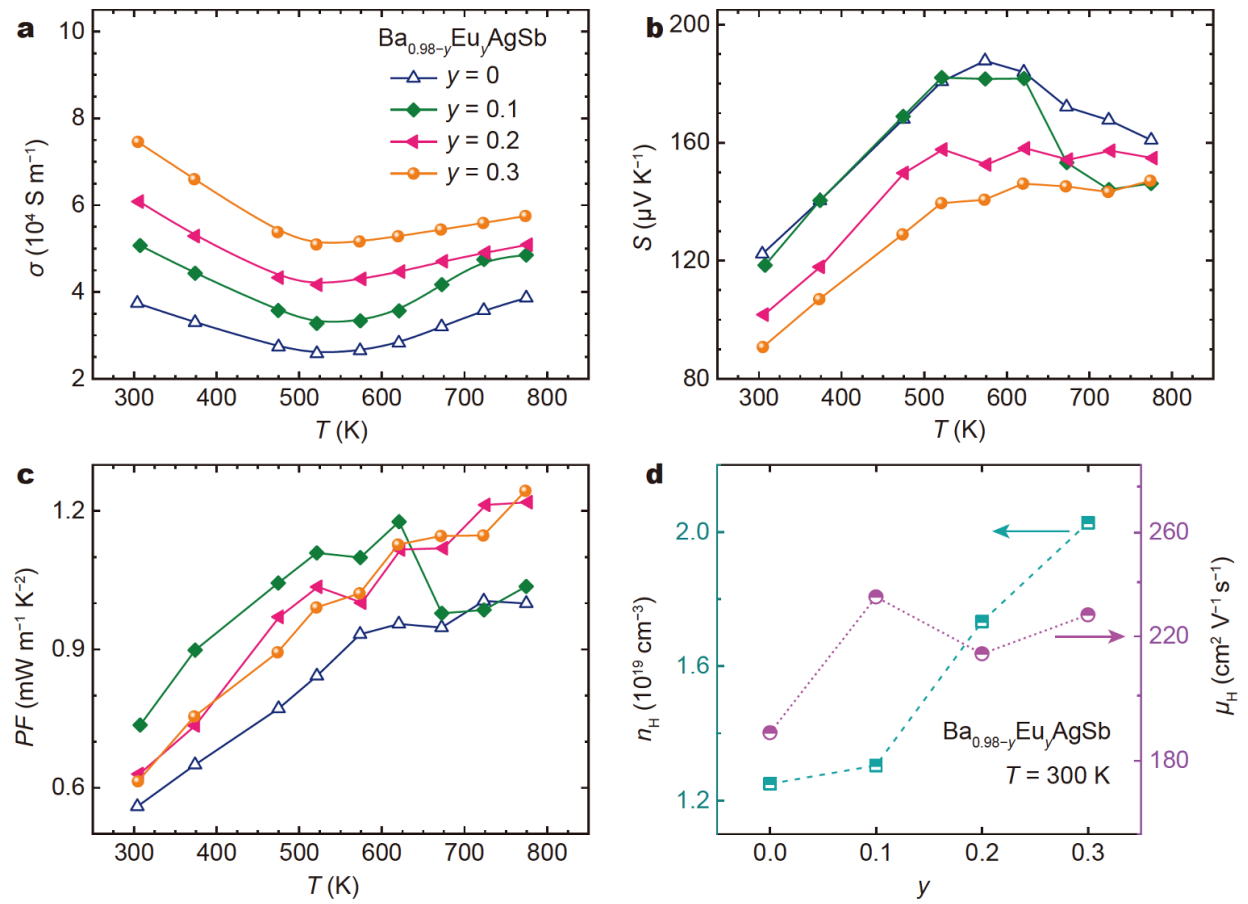

Figure 5 Temperature-dependent (a) electrical conductivity, (b) Seebeck coefficient, and (c) power factor of $\mathrm{Ba}_{0.98-y} \mathrm{Eu}_{y} \mathrm{AgSb}(y=0,0.1,0.2$, and 0.3). (d) Room-temperature Hall carrier concentration and mobility of $\mathrm{Ba}_{0.98-y} \mathrm{Eu}_{y} \mathrm{AgSb}(y=0,0.1,0.2$, and 0.3$)$.
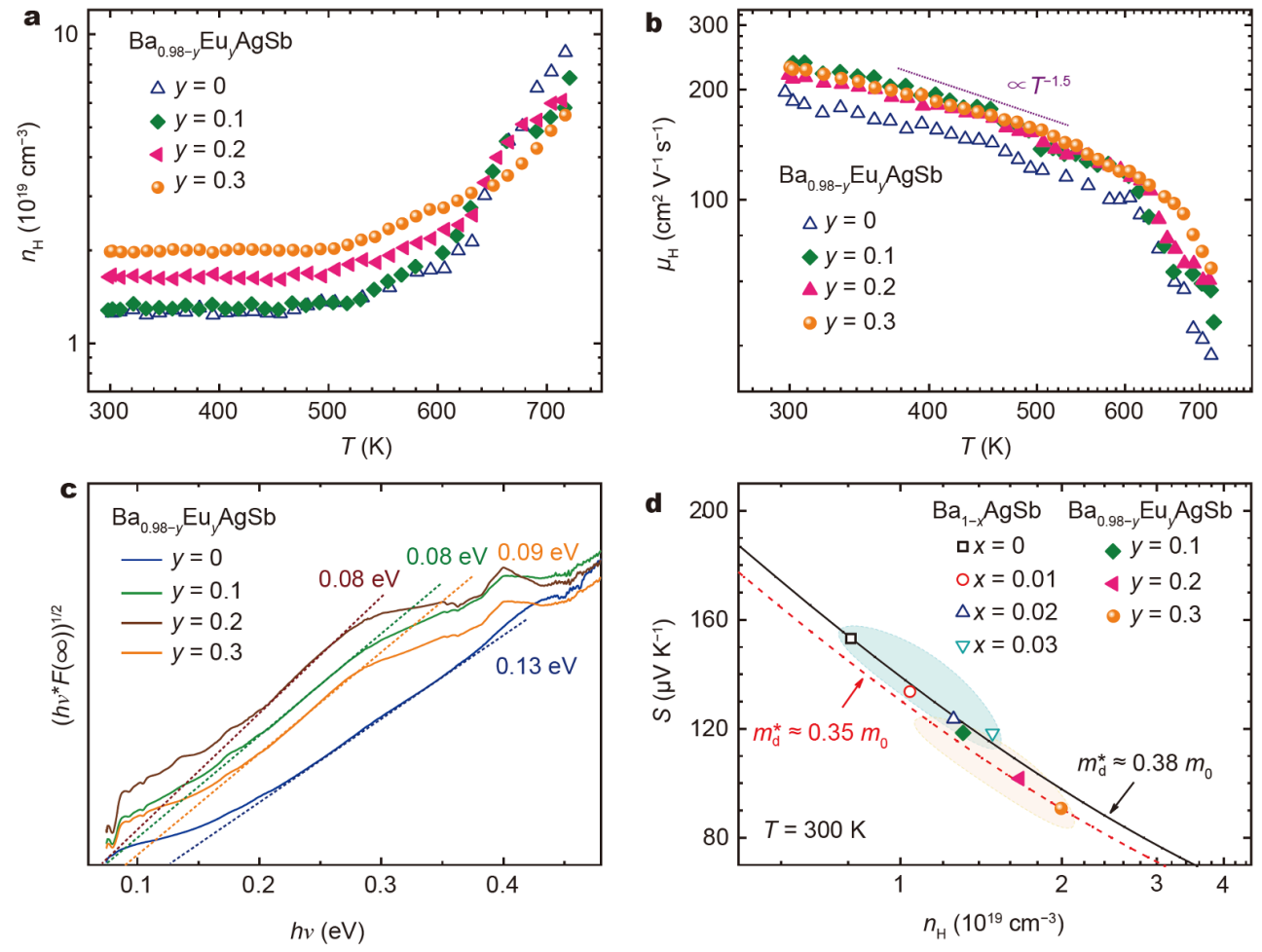

Figure 6 (a) Hall carrier concentration and (b) mobility of $\mathrm{Ba}_{0.98-y} \mathrm{Eu}_{y} \mathrm{AgSb}(y=0,0.1,0.2$, and 0.3$)$ as a function of temperature. (c) Normalized optical absorption versus photon energy for $\mathrm{Ba}_{0.98-y} \mathrm{Eu}_{y} \mathrm{AgSb}\left(y=0.1,0.2\right.$, and 0.3). (d) Room-temperature Pisarenko relations of $\mathrm{Ba}_{1-x} \mathrm{AgSb}(x=0$, $0.01,0.02$, and 0.03$)$ and $\mathrm{Ba}_{0.98-y} \mathrm{Eu}_{y} \mathrm{AgSb}(y=0.1,0.2$, and 0.3$)$. 
centration increases below the intrinsic excitation temperature because of the high carrier concentration of EuAgSb [33]. The carrier mobility tends to show a $\mu_{\mathrm{H}} \propto$ $T^{-1.5}$ relationship, implying that the transport scattering mechanism is dominated by phonon scattering. As shown in Fig. $6 \mathrm{c}$, the measured $E_{\mathrm{g}}$ of $\mathrm{Ba}_{0.98} \mathrm{AgSb}$ is $\sim 0.13 \mathrm{eV}$, and then the value decreases after Eu alloying. The roomtemperature Pisarenko relation of $\mathrm{Ba}_{1-x} \mathrm{AgSb}(x=0,0.01$, 0.02 , and 0.03$)$ and $\mathrm{Ba}_{0.98-y} \mathrm{Eu}_{y} \mathrm{AgSb}(y=0.1,0.2$, and 0.3$)$ is shown in Fig. 6d. The relationship of Seebeck coefficient and carrier concentration for $\mathrm{Ba}_{1-x} \mathrm{AgSb}$ matches well with the Pisarenko relation estimated by the SPB model. With the increase of Eu content, the effective mass decreases gradually from $\sim 0.38 m_{0} \quad\left(m_{0}\right.$ is electron mass) to $\sim 0.35 \mathrm{~m}_{0}$, which is believed to contribute to the enhanced $\mu_{\mathrm{H}}$. In addition, the disappearance of secondary phase also leads to the improvement of $\mu_{\mathrm{H}}$.

The temperature-dependent total thermal conductivity and lattice thermal conductivity are plotted in Fig. 7a, b, respectively. The room-temperature thermal conductivity of $\mathrm{Ba}_{0.98-y} \mathrm{Eu}_{y} \mathrm{AgSb}$ decreases from $\sim 2.5$ to $\sim 1.5 \mathrm{~W} \mathrm{~m}^{-1} \mathrm{~K}^{-1}$. In Fig. $7 \mathrm{~b}$, the lattice thermal conductivity is obviously reduced by Eu alloying. With the increasing Eu content, an approximate $\kappa_{\mathrm{L}} \propto T^{-0.5}$ relationship between the lattice thermal conductivity and temperature indicates that strong alloy scattering dominates [41]. In order to further describe the scattering mechanisms, we used the Callaway model to analyze the lattice thermal conductivity with phonon relaxation time under different scattering mechanisms [42]:

$$
\begin{aligned}
\kappa_{\mathrm{L}} & =\int_{0}^{\omega_{\mathrm{D}} / k_{\mathrm{B}} T} \kappa_{s}(\omega) \mathrm{d} \omega \\
& =\frac{k_{\mathrm{B}}}{2 \pi^{2} v}\left(\frac{k_{\mathrm{B}}}{\hbar}\right)^{3} T^{3} \int_{0}^{\theta_{\mathrm{D}} / T} \tau(x) \frac{x^{4} e^{x}}{\left(e^{x}-1\right)^{2}} \mathrm{~d} x,
\end{aligned}
$$

where $k_{\mathrm{B}}, v, \hbar, T, \theta_{\mathrm{D}}$, and $\tau$ are the Boltzmann constant, phonon velocity, reduced Planck constant, absolute temperature, Debye temperature, and phonon relaxation time, respectively, and $x$ is the reduced phonon frequency $x=\hbar \omega / k_{\mathrm{B}} T$ ( $\omega$ is the phonon frequency). The phonon relaxation time $\tau$ can be obtained by the addition of the inverse relaxation times for different scattering processes:

$\tau^{-1}=\tau_{\mathrm{U}}^{-1}+\tau_{\mathrm{N}}^{-1}+\tau_{\mathrm{B}}^{-1}+\tau_{\mathrm{PD}}^{-1}$,

where $\tau_{\mathrm{U}}, \tau_{\mathrm{N}}, \tau_{\mathrm{B}}$, and $\tau_{\mathrm{PD}}$ refer to the phonon relaxation time for Umklapp scattering, normal scattering, boundary
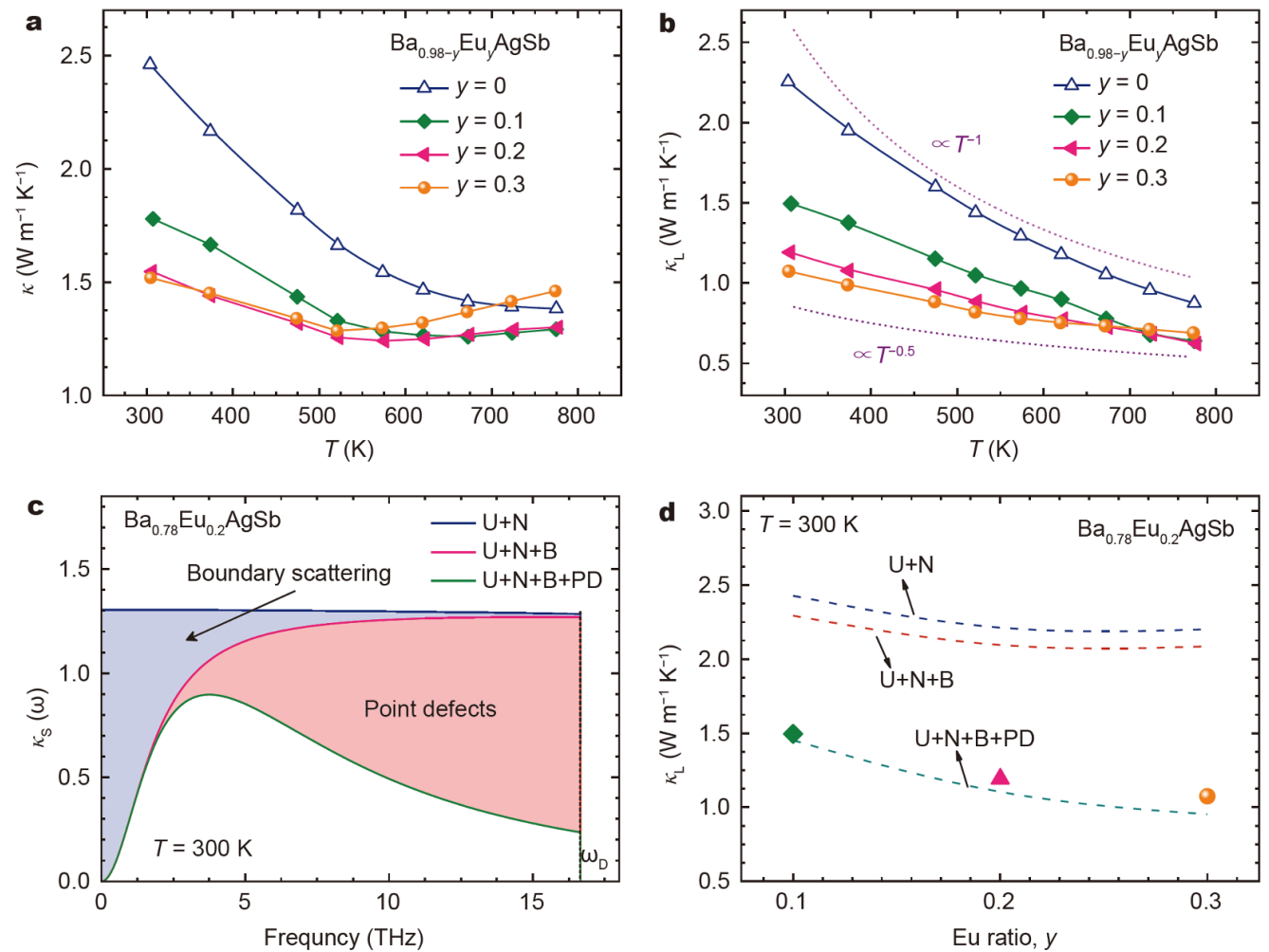

Figure 7 (a) Thermal conductivity and (b) lattice thermal conductivity of $\mathrm{Ba}_{0.98-y} \mathrm{Eu}_{y} \mathrm{AgSb}(y=0,0.1,0.2$, and 0.3$)$ as a function of temperature. (c) Frequency-dependent lattice thermal conductivity of $\mathrm{Ba}_{0.78} \mathrm{Eu}_{0.2} \mathrm{AgSb}$. (d) Room-temperature lattice thermal conductivity of $\mathrm{Ba}_{0.98-y} \mathrm{Eu}_{y} \mathrm{AgSb}$ as a function of $y$. 
scattering, and point defect scattering, respectively. Their detailed formulas can be found in the Supplementary information and the related publications [42-44]. Here the electron-phonon scattering was not considered because $\mathrm{Ba}_{0.98-y} \mathrm{Eu}_{y} \mathrm{AgSb}$ has much lower carrier concentrations than those of half-Heusler alloys [42]. Fig. 7c shows the frequency-dependent lattice thermal conductivity of $\mathrm{Ba}_{0.78} \mathrm{Eu}_{0.2} \mathrm{AgSb}$. The thermal conductivity decreased by grain boundary scattering is concentrated in the range of frequency $<5 \mathrm{THz}$ (blue region), while the thermal conductivity decreased by point defects is in the range of medium-to-high frequency (red region). The decreased lattice thermal conductivity in $\mathrm{Ba}_{0.98-y} \mathrm{Eu}_{y} \mathrm{AgSb}$ is mainly related to the point defects, as demonstrated in Fig. $7 \mathrm{~d}$.

The $Z T$ values of $\mathrm{BaAgSb}$ and $\mathrm{Ba}_{0.98-y} \mathrm{Eu}_{y} \mathrm{AgSb}(y=0$, $0.1,0.2$, and 0.3 ) are presented in Fig. 8. The point defects introduced by both Ba deficiency and Eu alloying are of great benefit to the thermoelectric properties of $\mathrm{BaAgSb}$. The peak $Z T$ of $\mathrm{Ba}_{0.78} \mathrm{Eu}_{0.2} \mathrm{AgSb}$ reaches $\sim 0.73$ at $773 \mathrm{~K}$, which is comparable to some typical Zintl-phase thermoelectric materials. This work enriches the family of Zintl-phase thermoelectric materials for the mid-temperature thermoelectric applications.

\section{CONCLUSION}

In summary, the intrinsic excitation in BaAgSb prevents the increase of $Z T$ at high temperatures. Ba deficiency can increase the carrier concentration, and lead to enhanced thermoelectric properties at high temperatures. A peak $Z T$ of $\sim 0.56$ was obtained in $\mathrm{Ba}_{0.98} \mathrm{AgSb}$ at $773 \mathrm{~K}$. Furthermore, Eu alloying at Ba site can not only lower the lattice thermal conductivity by inducing point-defect

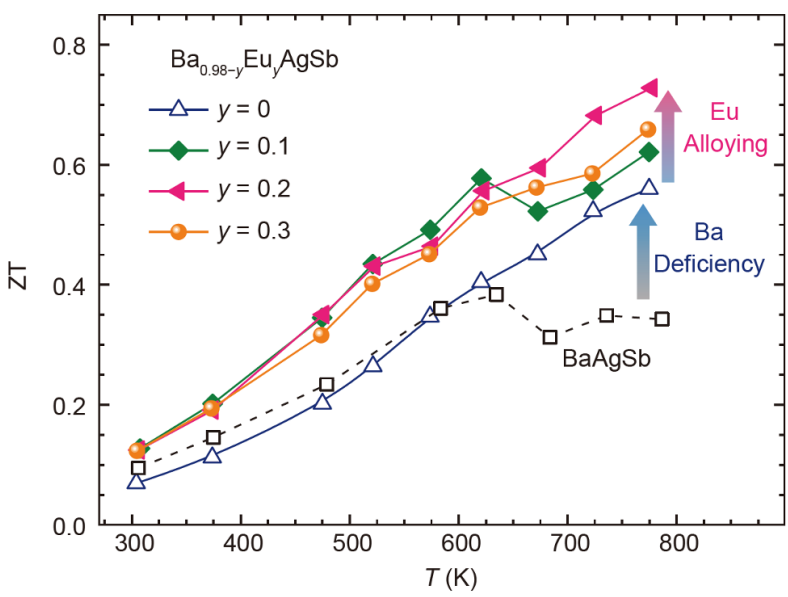

Figure 8 Temperature-dependent $Z T$ values of $\mathrm{BaAgSb}$ and $\mathrm{Ba}_{0.98-y} \mathrm{Eu}_{y} \mathrm{AgSb}(y=0,0.1,0.2$, and 0.3). scattering, but also improve the electrical properties by increasing the carrier mobility. Benefitting from the enhancement by two types of point defects, the $Z T$ value was improved from $\sim 0.34$ for $\mathrm{BaAgSb}$ to $\sim 0.73$ for $\mathrm{Ba}_{0.78} \mathrm{Eu}_{0.2} \mathrm{AgSb}$ at $773 \mathrm{~K}$. These results thus demonstrate that point defect engineering is promising for further optimizing Zintl-phase thermoelectric materials.

Received 26 November 2020; accepted 7 February 2021; published online 14 May 2021

1 Mao J, Liu Z, Zhou J, et al. Advances in thermoelectrics. Adv Phys, 2018, 67: 69-147

2 Liu W, Hu J, Zhang S, et al. New trends, strategies and opportunities in thermoelectric materials: A perspective. Mater Today Phys, 2017, 1: 50-60

3 Cai B, Hu H, Zhuang HL, et al. Promising materials for thermoelectric applications. J Alloys Compd, 2019, 806: 471-486

$4 \mathrm{Wu}$ Y, Nan P, Chen Z, et al. Thermoelectric enhancements in PbTe alloys due to dislocation-induced strains and converged bands. Adv Sci, 2020, 7: 1902628

5 Pei Y, Wang H, Snyder GJ. Band engineering of thermoelectric materials. Adv Mater, 2012, 24: 6125-6135

6 Liu X, Xi L, Qiu W, et al. Significant roles of intrinsic point defects in $\mathrm{Mg}_{2} \mathrm{X}(\mathrm{X}=\mathrm{Si}, \mathrm{Ge}, \mathrm{Sn})$ thermoelectric materials. Adv Electron Mater, 2016, 2: 1500284

7 Hu L, Zhu T, Liu X, et al. Point defect engineering of high-performance bismuth-telluride-based thermoelectric materials. Adv Funct Mater, 2014, 24: 5211-5218

8 Rogl G, Rogl P. How nanoparticles can change the figure of merit, ZT, and mechanical properties of skutterudites. Mater Today Phys, 2017, 3: 48-69

9 Mao J, Liu Z, Ren Z. Size effect in thermoelectric materials. npj Quant Mater, 2016, 1: 16028

10 Liu R, Chen H, Zhao K, et al. Entropy as a gene-like performance indicator promoting thermoelectric materials. Adv Mater, 2017, 29: 1702712

11 Hu L, Zhang Y, Wu H, et al. Entropy engineering of SnTe: Multiprincipal-element alloying leading to ultralow lattice thermal conductivity and state-of-the-art thermoelectric performance. Adv Energy Mater, 2018, 8: 1802116

12 Ohno S, Imasato K, Anand S, et al. Phase boundary mapping to obtain n-type $\mathrm{Mg}_{3} \mathrm{Sb}_{2}$-based thermoelectrics. Joule, 2018, 2: 141154

13 Li X, Yang P, Wang Y, et al. Phase boundary mapping in ZrNiSn half-Heusler for enhanced thermoelectric performance. Research, 2020, 2020: 4630948

14 Shuai J, Mao J, Song S, et al. Recent progress and future challenges on thermoelectric Zintl materials. Mater Today Phys, 2017, 1: 7495

15 Kauzlarich SM, Brown SR, Jeffrey Snyder G. Zintl phases for thermoelectric devices. Dalton Trans, 2007, 2099-2107

16 Liu KF, Xia SQ. Recent progresses on thermoelectric Zintl phases: Structures, materials and optimization. J Solid State Chem, 2019, 270: $252-264$

17 Zevalkink A, Zeier WG, Cheng E, et al. Nonstoichiometry in the Zintl phase $\mathrm{Yb}_{1-\delta} \mathrm{Zn}_{2} \mathrm{Sb}_{2}$ as a route to thermoelectric optimization. Chem Mater, 2014, 26: 5710-5717 
18 Ohno S, Aydemir U, Amsler M, et al. Achieving $\mathrm{zT}>1$ in inexpensive Zintl phase $\mathrm{Ca}_{9} \mathrm{Zn}_{4+x} \mathrm{Sb}_{9}$ by phase boundary mapping. Adv Funct Mater, 2017, 27: 1606361

19 Zheng L, Li W, Wang X, et al. Alloying for orbital alignment enables thermoelectric enhancement of $\mathrm{EuCd}_{2} \mathrm{Sb}_{2}$. J Mater Chem A, 2019, 7: 12773-12778

20 Shuai J, Kim HS, Liu Z, et al. Thermoelectric properties of Zintl compound $\mathrm{Ca}_{1-x} \mathrm{Na}_{x} \mathrm{Mg}_{2} \mathrm{Bi}_{1.98}$. Appl Phys Lett, 2016, 108: 183901

21 Zheng L, Li W, Sun C, et al. Ternary thermoelectric $\mathrm{AB}_{2} \mathrm{C}_{2}$ Zintls. J Alloys Compd, 2020, 821: 153497

22 Zhu M, Wu Z, Liu Q, et al. Defect modulation on $\mathrm{CaZn}_{1-x} \mathrm{Ag}_{1-y} \mathrm{Sb}$ $(0<x<1 ; 0<y<1)$ Zintl phases and enhanced thermoelectric properties with high $z T$ plateaus. J Mater Chem A, 2018, 6: 1177311782

23 Wang $\mathrm{Y}$, Bobev $\mathrm{S}$. Rare-earth metal substitutions in $\mathrm{Ca}_{9_{-x}} \mathrm{RE}_{x} \mathrm{Mn}_{4^{-}}$ $\mathrm{Sb}_{9}(\mathrm{RE}=\mathrm{La}-\mathrm{Nd}, \mathrm{Sm} ; x \approx 1)$. synthesis and characterization of a new series of narrow-gap semiconductors. Chem Mater, 2018, 30: 3518-3527

24 Shuai J, Wang Y, Liu Z, et al. Enhancement of thermoelectric performance of phase pure Zintl compounds $\mathrm{Ca}_{1-x} \mathrm{Yb}_{x} \mathrm{Zn}_{2} \mathrm{Sb}_{2}$, $\mathrm{Ca}_{1-x} \mathrm{Eu}_{x} \mathrm{Zn}_{2} \mathrm{Sb}_{2}$, and $\mathrm{Eu}_{1-x} \mathrm{Yb}_{x} \mathrm{Zn}_{2} \mathrm{Sb}_{2}$ by mechanical alloying and hot pressing. Nano Energy, 2016, 25: 136-144

25 Zhang Z, Yan Y, Li X, et al. A dual role by incorporation of magnesium in $\mathrm{YbZn}_{2} \mathrm{Sb}_{2}$ Zintl phase for enhanced thermoelectric performance. Adv Energy Mater, 2020, 10: 2001229

26 Wang $\mathrm{X}, \mathrm{Li}$ J, Wang $\mathrm{C}$, et al. Orbital alignment for high performance thermoelectric $\mathrm{YbCd}_{2} \mathrm{Sb}_{2}$ alloys. Chem Mater, 2018, 30: 5339-5345

27 Chen C, Xue W, Li X, et al. Enhanced thermoelectric performance of Zintl phase $\mathrm{Ca}_{9} \mathrm{Zn}_{4+x} \mathrm{Sb}_{9}$ by beneficial disorder on the selective cationic site. ACS Appl Mater Interfaces, 2019, 11: 37741-37747

$28 \mathrm{Wu} \mathrm{Z}$, Li J, Li X, et al. Tuning the thermoelectric properties of $\mathrm{Ca}_{9} \mathrm{Zn}_{4+x} \mathrm{Sb}_{9}$ by controlled doping on the interstitial structure. Chem Mater, 2016, 28: 6917-6924

29 Toberer ES, Zevalkink A, Crisosto N, et al. The Zintl compound $\mathrm{Ca}_{5} \mathrm{Al}_{2} \mathrm{Sb}_{6}$ for low-cost thermoelectric power generation. Adv Funct Mater, 2010, 20: 4375-4380

30 Toberer ES, Cox CA, Brown SR, et al. Traversing the metalinsulator transition in a Zintl phase: Rational enhancement of thermoelectric efficiency in $\mathrm{Yb}_{14} \mathrm{Mn}_{1-x} \mathrm{Al}_{\mathrm{x}} \mathrm{Sb}_{11}$. Adv Funct Mater, 2008, 18: 2795-2800

31 Chen $\mathrm{C}$, Xue W, Li S, et al. Zintl-phase $\mathrm{Eu}_{2} \mathrm{ZnSb}_{2}$ : A promising thermoelectric material with ultralow thermal conductivity. Proc Natl Acad Sci USA, 2019, 116: 2831-2836

32 Merlo F, Pani M, Fornasini ML. RMX compounds formed by alkaline earths, europium and ytterbium-I. Ternary phases with $\mathrm{M} \equiv \mathrm{Cu}, \mathrm{Ag}, \mathrm{Au} ; \mathrm{X} \equiv \mathrm{Sb}$, Bi. J Less Common Met, 1990, 166: 319_ 327

33 Zhang W, Chen C, Yao H, et al. Promising Zintl-phase thermoelectric compound SrAgSb. Chem Mater, 2020, 32: 6983-6989

34 Chen $\mathrm{C}$, Li X, Xue W, et al. Manipulating the intrinsic vacancies for enhanced thermoelectric performance in $\mathrm{Eu}_{2} \mathrm{ZnSb}_{2}$ Zintl phase. Nano Energy, 2020, 73: 104771

35 Perdew JP, Burke K, Ernzerhof M. Generalized gradient approximation made simple. Phys Rev Lett, 1996, 77: 3865-3868

36 Blöchl PE. Projector augmented-wave method. Phys Rev B, 1994, 50: $17953-17979$

37 Chanakian S, Uhl D, Neff D, et al. Exceptionally high electronic mobility in defect-rich $\mathrm{Eu}_{2} \mathrm{ZnSb}_{2-x} \mathrm{Bi}_{x}$ alloys. J Mater Chem A, 2020, 8: 6004-6012
38 Zhang S, Wang Q, Kawazoe Y, et al. Three-dimensional metallic boron nitride. J Am Chem Soc, 2013, 135: 18216-18221

39 Sun X, Li X, Yang J, et al. Achieving band convergence by tuning the bonding ionicity in n-type $\mathrm{Mg}_{3} \mathrm{Sb}_{2}$. J Comput Chem, 2019, 40: 1693-1700

40 Thesberg M, Kosina H, Neophytou N. On the Lorenz number of multiband materials. Phys Rev B, 2017, 95: 125206

41 Liu $\mathrm{X}$, Zhu $\mathrm{T}$, Wang $\mathrm{H}$, et al. Low electron scattering potentials in high performance $\mathrm{Mg}_{2} \mathrm{Si}_{0.45} \mathrm{Sn}_{0.55}$ based thermoelectric solid solutions with band convergence. Adv Energy Mater, 2013, 3: 12381244

42 Xie $\mathrm{H}$, Wang H, Pei Y, et al. Beneficial contribution of alloy disorder to electron and phonon transport in half-Heusler thermoelectric materials. Adv Funct Mater, 2013, 23: 5123-5130

43 Yang J, Meisner GP, Chen L. Strain field fluctuation effects on lattice thermal conductivity of $\mathrm{ZrNiSn}$-based thermoelectric compounds. Appl Phys Lett, 2004, 85: 1140-1142

44 Chen Z, Jian Z, Li W, et al. Lattice dislocations enhancing thermoelectric $\mathrm{PbTe}$ in addition to band convergence. Adv Mater, 2017, 29: 1606768

Acknowledgements This work was supported by the National Natural Science Foundation of China (51871081, 11674078, 51971081, and 52001339), the Cheung Kong Scholar Reward Program Young Scholar Program of China (Q2018239), the Natural Science Foundation of Guangdong Province of China (2018A0303130033), the Natural Science Foundation for Distinguished Young Scholars of Guangdong Province (2020B1515020023), Shenzhen Science and Technology Innovation Plan (KQJSCX20180328165435202 and JCYJ20180307154619840), and the National Key Research and Development Program of China (2017YFA0303600).

Author contributions Chen Y, Cao F, and Zhang Q conceived the project; Huang $\mathrm{Y}$, Chen $\mathrm{C}$, Zhang $\mathrm{W}$, and $\mathrm{Li} \mathrm{X}$ prepared the samples; Huang $Y$, Liu $Y$, Wang $X$, and Zhang $Z$ performed the property measurements; Xue W performed the TEM measurement; Yao H conducted the DFT calculation; Wang Y, Liu X, Cao F, and Zhang Q contributed new reagents/analytic tools; Chen C, Huang Y, Wang Y, Chen Y, Cao F, and Zhang $\mathrm{Q}$ analyzed the data and wrote the paper.

Conflict of interest The authors declare that they have no conflict of interest.

Supplementary information Supporting data are available in the online version of the paper.

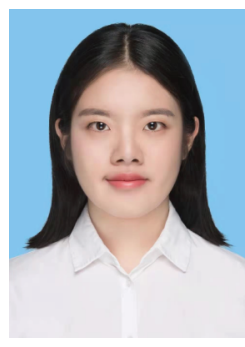

Yifang Huang received her Bachelor degree in materials science and engineering from Harbin Institute of Technology (Shenzhen) in 2020. She is currently a Master's degree candidate at Shanghai Jiao Tong University. Her research interest mainly focuses on thermoelectric materials. 


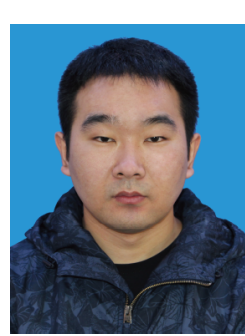

Chen Chen is currently a research associate at the Department of Mechanical Engineering, University of Hong Kong (HKU). He received his $\mathrm{PhD}$ degree from the Department of Materials Science and Engineering, Yanshan University in 2017 . He was a postdoctoral fellow at the School of Materials Science and Engineering, Harbin Institute of Technology (Shenzhen) (2017-2020). His current research mainly focuses on improving the thermoelectric performance of Zintl-phase compounds.

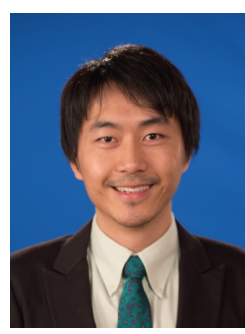

Yue Chen is an associate professor at the Department of Mechanical Engineering, HKU. His research interests focus on the materials physics for electrical and thermal transports, such as electronic structures and lattice dynamics. His interests stem from the studies of materials science at Oxford University and Beihang University. He was a postdoctoral fellow at Columbia University in the City of New York before joining HKU.

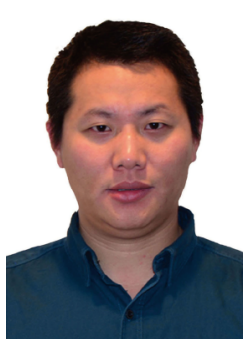

Feng Cao is currently an associate professor at the School of Science at Harbin Institute of Technology (Shenzhen). He received his $\mathrm{PhD}$ degree in physics from The Hong Kong Polytechnic University in 2012, followed by the postdoctoral appointments from Boston College and University of Houston. His current research focuses on the design of thermoelectric devices for waste heat recovery and the optical functional films for tuning solar spectrum.

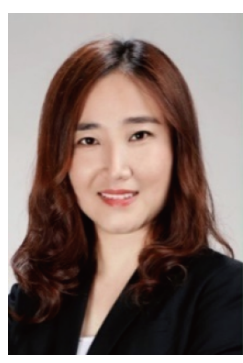

Qian Zhang is currently a professor at the School of Materials Science and Engineering, Harbin Institute of Technology (Shenzhen). She obtained her PhD degree from Zhejiang University. After that, she spent a year as a postdoctoral fellow at the National University of Singapore. She was a research associate at Boston College and University of Houston. Her current research focuses on enhancing the existing thermoelectric materials and discovering new thermoelectric materials for waste heat recovery.

\section{利用点缺陷策略提高Zintl相BaAgSb的热电性能}

黄奕芳 ${ }^{1 \dagger}$, 陈辰 ${ }^{1,2 \dagger}$, 张伟明 ${ }^{1}$, 李孝芳 ${ }^{1}$, 薛文华 ${ }^{1,3}$, 王心宇 ${ }^{1}$, 刘一杰 ${ }^{4}$, 姚洪浩 ${ }^{1}$, 张宗委 ${ }^{1}$, 陈奥 ${ }^{2 *}$, 曹峰 ${ }^{*}$, 刘兴军 ${ }^{1,5}$, 王玉梅 ${ }^{3}$, 张倩 ${ }^{1,5^{*}}$

摘要 Zintl相化合物由于其独特的“声子玻璃-电子晶体”(PGEC)结 构在热电领域具有广泛的应用前景. 本文报道了Zintl相热电材料 $\mathrm{BaAgSb}$ 的热电性质. 通过引入 $\mathrm{Ba}$ 空位, 可以提高 $\mathrm{p}$ 型 $\mathrm{BaAgSb}$ 的载流 子浓度, 抑制其本征激发, $\mathrm{Ba}_{0.98} \mathrm{AgSb}$ 的 $Z T$ 值在 $773 \mathrm{~K}$ 达到了 0.56. 此外, 在 $\mathrm{Ba}$ 位进一步合金化 $\mathrm{Eu}$, 不仅能够引入点缺陷散射降低晶格 热导率, 而且由于载流子迁移率的提高使其电输运性质也得到了 改善. 最终, $\mathrm{Ba}_{0.78} \mathrm{Eu}_{0.2} \mathrm{AgSb}$ 的 $Z T$ 值在 $773 \mathrm{~K}$ 达到 $~ 0.73$. 